\title{
Time Processing in Schizophrenia
}

\author{
Deana B. Davalos * and Jamie Opper*
}

Time is inside as well as outside of ourselves. Time is a perception. It is part of the outside world, but it is also a sensation immediately experienced in ourselves. We organize and crystalize the perception of time into the connotation of a continuous flowing time, which we measure by clocks, and we try to apply the same measures to the time experience in ourselves, to what we may call time sensation.... Time is an inherent part of the world of perception, outside and inside the body.

(SCHILDER, 1936)

Schilder (1936) described his conception of time in his paper, Psychopathology of Time, which addressed time perception and the idea that various types of psychopathology involved a disturbance in time perception. Schilder wrote the paper while at Bellevue Psychiatric Hospital and detailed a variety of disorders and the possible temporal distortion associated with each type of pathology. About schizophrenia, he quotes a patient who says, “I can't orient myself in the world - I am not clear anymore...I continue to live in eternity. There is no hour, no noon, no night.... Time does not move. I am wavering between past and future." Schilder's observations of timing dysfunction in his schizophrenic patients led him to ask, “...why does the schizophrenic give up his time experience? What does time mean for him?" The questions regarding time perception and the role timing plays in schizophrenia continue to perplex researchers today. Our understanding of time processing in schizophrenia has developed over the years from a once rather simplistic view of temporal dysfunction to an elaborate organization of temporal deficits that span from simple sensory measures of timing to higher order processes. And while it has been argued for decades that time processing is disrupted in schizophrenia, the breadth of the implications stemming from those temporal deficits have grown to include areas of clinical symptomatology, social and emotional processing, language, to higher order cognitive processes.

* Colorado State University, USA.

(C) DEANA B. DAVALOS \& JAMIE OPPER, 2015 | DOI 10.1163/9789004230699_005 This is an open access chapter distributed under the terms of the Creative Commons Attribution-Noncommercial 3.0 Unported (CC-BY-NC 3.0) License, 
Conceptually, our capacity to process time is viewed as an ability that plays a critical role in our perception of the world around us. Navon (1978) wrote that our perception of the world consists of a hierarchy of dimensions and that time is at the top of that hierarchy. The ability to process time has been associated with relatively basic tasks such as planning and sequencing and processing basic sensory input to higher order processes that are involved in athletic ability, driving, language, walking, and musical ability (Eagleman et al., 2005; Eagleman, 2009; Ferrandez et al., 2003; Macar et al., 2006; Mangels et al., 1998; Tracy et al., 1998). Given the widespread collection of behaviors, actions, and cognitive processes that appear to be influenced by timing, investigators have begun to speculate whether deficits in information processing and higher level cognitive processing that have been associated with dysexecutive syndrome, and schizophrenia specifically, may be in part due to temporal dysfunction (Macar and Vidal, 2009; Volz et al., 2001). And while time processing may not have a place in the scientific literature as extensive as other cognitive processes, such as attention, working memory, or inhibition, there are indicators that suggest that the magnitude of the importance of intact temporal processing is beginning to be understood. First, Head and colleagues (2008) have recently included time processing as one of the few possible "cognitive primitives" or what is described as basic neuropsychological processess that have broad influence on other cognitive functions, but cannot be separated into component processes themselves (Salthouse, 1985; Verhaeghen and Salthouse, 1997; Zacks and Hasher, 1994). While Head et al. have introduced the idea that time perception has a place in the hierarchy of cognitive processes alongside what have been viewed as fundamental cognitive capacities, such as inhibition and processing speed, there have been relatively few studies that can be used to support the argument. Cognitive primitives cannot, by definition, be separated into component processes and many of the current studies assessing time processing highlight the complexity of measuring temporal processing as most timing tasks generally involve some degree of attentional resources, decision making, and vigilance. Head's proposal, nonetheless, highlights the fact that timing is now considered as being a foundational cognitive process that may be involved in widespread dysfunction. The idea that temporal processing may lead to some type of dysfunction highlights the second indicator that stresses the magnitude of the importance of intact time processing.

The scientific literature is rich with studies assessing clinical populations and how time processing may be at the root of clinical symptomatology and deficits associated with various disorders. While it is beyond the scope of this chapter to address all of the types of psychopathology associated with temporal processing, included in the list of clinical disorders and/or clinical features 
are: aphasia, Alzheimer's disease, attention-deficit hyperactivity disorder, dyslexia, apraxia of speech, traumatic brain injury, autism-spectrum disorders, and schizophrenia (Anderson and Schmitter-Edgecombe, 2011; Barabasz, 1973; Bauermeister et al., 2005; Carroll, O'Donnell, Shekhar, and Hetrick, 2009; Condray, 2005; Efron, 1963; Kaminsky et al., 2002; Kraus, 2003; Merzenich et al., 1996; Rueda and Schmitter-Edgecombe, 2009; Wallace and Happe, 2008; Ziegler and von Cramon, 1986). These represent but a few of the many clinical disorders that are linked to time processing deficits. The breadth of disorders that link clinical symptomatology to temporal processing difficulties suggest a critical role between timing and clinical health, or more specifically clinical psychopathology. Regarding schizophrenia, the link between the disorder and timing dysfunction has been recognized for nearly a century. Minkowski (1931) argued that many clinical disorders could be linked to a disorder in space, time, or memory. Specifically, he described conditions such as dementia as being disturbed in terms of memory while the space and time "I-Here-Now" factors are intact. Schizophrenia, on the other hand, appears to involve intact memory, with disturbed "I-Here-Now" factors (Minkowski, 1928). Israeli (1932) eloquently reviews physician and researchers' perspectives on time processing in schizophrenia during the 1920's and 1930's and highlights the personal views of distorted time in patients with schizophrenia. Specifically, he discusses patient complaints presented by Fischer (1929, 1930a,b,c), which stress the "I-HereNow" disturbance. Fischer reports that his patients complain of: feeling that time has come to a standstill which is compensated for by a dreamlike existence, demonic playing with time, praying for the destruction of time, and feelings of timelessness. Fischer goes on to speculate that there is no symptom of schizophrenia, which is not a product of a time-space disturbance.

The idea that most symptoms of schizophrenia, both cognitive and clinical, may be affected by a disturbance in timing has persisted over the years. Eagleman and Holcombe (2002) point to research, which argues that individuals with schizophrenia may believe that their own thoughts are caused by someone else or possess a delusion that unrelated events are attributable to their actions (Frith, Blakemore, and Wolpert, 2002). In Frith and colleagues' research, they speculate that these disturbances may be due to a lack of awareness of certain aspects of motor control. Eagleman and Holcombe have argued that rather than an emphasis placed on difficulties in the processing of motor control, these symptoms could instead be due to difficulties in temporal processing. Stetson et al. (2006) have shown that when healthy control subjects had a relatively simple sensory-motor task slightly adjusted in terms of the interval duration between when a button was pushed and a stimulus was presented, it induced a sense of not being in control of their physical response. 
Specifically, participants were first asked to simply press a button, which instantly resulted in a nearby light to flash. The paradigm was then slightly changed to add a tenth of a second delay between the button press and the flash of light appearing. Following this condition with the slight delay, the experimenters removed the delay and returned to the initial presentation. The removal of the delay appeared to induce in the participants, a perception that the flash was occurring before the responder had pressed the key. This study, among others, has led to questions about whether individuals with schizophrenia may experience was has been termed credit misattribution, or difficulty recognizing actions as one's own. The thought is that a relatively brief temporal distortion may be associated with the delusion that someone other than the individual themselves is in charge of their behavior (Gandhi, Wassef, and Eagleman, 2007). These findings of slight temporal distortion leading to delusions or feelings of confusion in healthy young adults has been compelling evidence in support of an argument for the important role temporal dysfunction may play in the clinical pathology of schizophrenia.

While research continues to explore the role of temporal deficits in schizophrenia, our understanding of the neural underpinnings of temporal dysfunction remains elusive. Comprehending the neural mechanisms involved in timing is important for a number of reasons. Given the breadth of timing deficits observed in schizophrenia, one question that persists is whether there may be a specific type of neural dysfunction that affects a wide range of processes or whether there are multiple areas of dysfunction in the schizophrenic brain that each contribute a unique piece to the various types of timing dysfunction observed.

To understand the complexity of trying to unravel the neural underpinnings of temporal processing, one first has to appreciate the plethora of tasks that are subsumed under the heading of time processing. The types of task that are considered timing-related represent a diverse set of processes ranging from time perception of durations in milliseconds, time-based prospective memory, temporal reproduction, temporal order, to temporal estimation of hours. Many researchers have attempted to organize types of temporal tasks to better understand timing. This need to clarify temporal processes, specifically in the context of psychopathology, is clear in the literature as early as the 1960 's. Lehmann (1967) presents one of the important distinctions in our understanding of time processing. He argues that there are two types of time, external time and internal time. External time is the "objective, universal, and absolute" time that is measured within a "conceptual framework." Internal time, on the other hand, is "subjective, individual, and relative" and he describes it as "the perception of enduring." It is this internal time that has been of most interest in the study of schizophrenia. Specifically, it is thought that disruptions in 
one's internal clock, even slight disruptions, appear to lead distortions in how individuals with schizophrenia conceive their environment and how they operate in their environment. And it is difficulties with the internal clock that appear to be at the root of a wide spectrum of clinical pathology in schizophrenia (e.g., hallucinations, delusions, psychomotor poverty, poverty of speech; Andreasen, 1999; Hyde, Ziegler, and Weinberger, 1993; McGlashan and Hoffman, 200o). In addition, temporal processing deficits have been argued by many to also affect cognitive functioning in schizophrenia.

For decades, researchers have posited that individuals with schizophrenia appear to process time differently than those who are not affected with the mental illness on a variety of behavioral and cognitive measures (Rabin, 1957). These deficits are noted on tasks assessing various types of time processing, including temporal perception, temporal estimation, and time reproduction (Davalos, Kisley, and Ross, 2002, 2003a; Rammsayer, 1990; Tysk, 1990). And these deficits of time processing seem to be widespread, spanning both auditory and visual temporal processing tasks (Schwartz, Mallott, and Winstead, 1988; Tysk, 1990). As will be addressed repeatedly in this Chapter, one of the primary limitations to understanding the neural underpinnings of time processing per se is the degree of nontemporal information that has historically been included in temporal processing tasks. Poynter and Homa (1983) point out that temporal perception tasks generally require the participant to be able to attend successfully to the nontemporal information that is often embedded in tasks. Given that most cognitive measures of timing employ attention and working memory, it is likely "temporal processing performance" in actuality reflects an amalgamation of multiple cognitive skills. The variations in attentional demands or recruitment of other cognitive resources have likely contributed to the variability in findings across behavioral studies and neurophysiology studies.

Mismatch negativity (MMN) has been utilized as a physiological measure of temporal processing. MMN is a brain response elicited to infrequent deviant stimuli in the context of recurring standard stimuli (Naatanen, 1992). One of the most important aspects of MMN is that it is generated in the absence of, or with minimal demands, on focused attention. Also, there is no motor response required nor is there a decision making element that is often involved in cognitive tasks and even other event related potentials (Sussman, Winkler, and Wang, 2003). MMN has been utilized in studies with a variety of clinical 
disorders, specifically those with suspected frontal deficits as it allows detection of deficits associated with frontal and temporal lobe dysfunction while minimizing confounding deficits associate with attention, vigilance, motivation, and decision making in these populations. MMN represents the neural process of detecting change in stimuli and generally exhibits maximal amplitude at frontal and central sites of the scalp. The main generators are thought to be located in the auditory and frontal cortices (Naatanen, 1992; Pulvermuller and Shtyrov, 2003). The frontal contribution to this waveform is thought to reflect an involuntary switch of attention to specific changes in the sensory environment (Deouell, Bentin, and Giard, 1998). MMN studies focusing on individuals with schizophrenia have included deviations in pitch, intensity, frequency, or, more specific to the current discussion, deviations in the duration of tones and/or interstimulus intervals (Baldeweg et al., 2002; Naatanen et al., 1993; Todd, Michie, and Jablensky, 2003). MMN paradigms utilizing duration intervals are thought to be valid representations of time processing as MMN amplitude is proportional to the relative discriminability of interval deviations, suggesting that the preattentive neural activity measured during M MN is that which is subsequently available for the conscious perception of time (Kisley et al., 2004). In schizophrenia, results consistently find that individuals with schizophrenia exhibit deficits in temporal processing measured via MMN (Baldeweg et al., 2002; Davalos, Kisley, and Ross, 2003b; Light and Braff, 2005). Light and Braff (2005a) have emphasized the practical applications of these deficits by linking these time-based MMN deficits to functional status (i.e., GAF Scale ratings and level of independence in community living situation). In their studies, an MMN utilizing a subtle temporal deviant (90\% standard, $50 \mathrm{msec}$, and $10 \%$ deviant, $100 \mathrm{msec}$, duration stimuli) was assessed in patients with schizophrenia. MMN deficits in this population were accounted for up to $42 \%$ of the variance in functional status. As the authors note, the correlation between time-based MMN deficits and impaired functioning in their study was observed as being much higher than the correlation between traditional neurocognitive measures and functional status and outcome (Green, 1996; Green et al., 2000; Palmer et al., 2002). In a separate study, the authors extend their findings by proposing that time-based MMN may also serve as a useful method to track progressive changes in neural substrate dysfunction and functional impairments over the course of schizophrenia (Light and Braff, 2005b). Light and Braff bolster this argument by highlighting past research indicating that while clinically unaffected relatives of individuals with schizophrenia and those with chronic schizophrenia exhibit mismatch negativity deficits, firstepisode patients exhibit intact mismatch negativity (Light and Braff, 2005b, Michie et al., 2002; Salisbury et al., 2002a; Umbricht et al., 2003). These findings 
support the utility of using temporal MMN indices as a tool for tracking brainbased changes associated with the progression of schizophrenia and possibly the role that MMN may play in serving as a biomarker for schizophrenia. While Light and Braff have highlighted the potential clinical utility of MMN, the authors point out that further research is needed to fully appreciate the potential strengths and limitations of time-based MMN in diagnoses and understanding of progression of schizophrenia. They argue that greater clarification is needed to understand the longitudinal course of time-based MMN deficits and the role that temporal processing measured via MMN may play in understanding neurodegeneration in schizophrenia.

It should be noted that if the reader questions whether it is the general electrophysiological index, MMN, independent of type of deviant, that appears to be the basis for the relationship between neurocognitive functional, functional status, and tracking progression of the disease in schizophrenia, the literature suggests otherwise. Specifically, past studies suggest that there is something unique about temporal deviants that are not present with other types of deviants. MMN response to duration deviants has been compared to frequency and intensity in the past to assess test-retest reliability and duration only was most replicable amplitude and latency among the deviances assessed. The authors argue then that duration-dependent MMN may be the best measure to utilize in the study of cognitive brain functioning (Tervaniemi et al., 1999). In general, these findings, along with others, illustrates the role that temporal processing measured via MMN plays in detecting vulnerability for schizophrenia, indirectly assessing the state of NMDA receptor functioning, and measuring the integrity of frontotemporal brain systems.

It should be noted, however, that recent electrophysiological research suggests that the timing deficits observed in schizophrenia are not generalized across all timing paradigms nor are they apparent across all areas of the brain. Rather, poor temporal processing was only noted for the conditions in which the difference between the standard interstimulus interval and the deviant interstimulus interval was more subtle (e.g., $15 \%$ different rather than $50 \%$ different; Davalos, Kisley, and Freedman, 2005). What was notable during the study was that there was a dissociation between behavioral performance and neurophysiological indices of temporal processing in the schizophrenia group. While their neurophysiological response was comparable to controls on the "easy" deviant, their behavioral performances were impaired compared to controls on both the "easy" and "difficult" deviants. The finding suggesting differences between conditions (easy vs. difficult) and across method of assessment (event-related potentials versus behavioral) suggests that the role of attention, motivation, and other mediating cognitive factors cannot be minimized when 
examining behavioral indices of time processing in populations that may be more affected by impairments in these cognitive areas. Salisbury et al. have argued in the past that the use of traditional behavioral measures of cognition paired with electrophysiological measures of brain activity allow for a unique multifaceted examination of behavior and the underlying processes (Salisbury et al., 2002b). And while ERP measures allow us to begin to understand that there are neurophysiological differences in the brains of patients with schizophrenia and healthy controls when it comes to temporal processing, it has not told us about the 'where' and 'how' of these processes.

\section{Functional Imaging/Localization}

The links between neurophysiology and behavioral indices of temporal processing highlight that there are clearly shared neural networks between both types of measures of time processing as well as distinct processes involved. This has led to questions about the neural etiology of time processing. Is there truly an "internal clock" that may be associated with all temporal information (Mangels et al., 1998)? And is this impaired internal clock the common link that is shared across an array of clinical disorders, such as PD, amnesia, cerebellar, and basal ganglia insult, in addition to frontal syndromes (Artieda et al., 1992; Casini and Ivry, 1999; Harrington and Haaland, 1998; Ivry and Keele, 1989; Nichelli et al., 1995; Rammsayer, 1993). Or is it more likely that there are specific types of temporal processing that rely on different neural networks?

For years it has been argued that the cerebellum is the key structure associated with short-duration processing while longer duration processing may be dependent on prefrontal cortical functioning (Clarke et al., 1996; Heatherington et al., 2000; Mangels, Ivry, and Shimizu, 1998). While the cerebellar argument appears to be relatively well established in terms of the role in automatic shortduration processing, the processing of longer durations appears more complex, with the relative roles of the basal ganglia and frontal cortex still contested (Hazeltine, Helmuth, and Ivry, 1997; Ivry, 1996). Specifying the neural underpinnings to processing longer durations is inherently more complicated because of the possible recruitment of other cognitive processes. Mangels et al. (1998) point out that longer durations (e.g., 3 or 4 s) utilized in interval comparison tasks may require working memory to keep those interval durations available for comparison. These types of temporal judgments may be particularly sensitive to frontal lobe functioning. Nichelli et al. (1995) supports this argument, noting that timing beyond 2 or $3 \mathrm{~s}$ may exceed more automatic "motor routines" and may require sustained attention and/or strategy use. Whether one argues for the necessity of sustained attention or working 
memory, it is likely that, unlike short intervals, longer intervals recruit the prefrontal cortex. Given the well-established prefrontal dysfunction associated with schizophrenia, this potential confound has made measuring the "true" neural correlates of temporal process difficult (Goldman-Rakic and Selemon, 1997; Goldman-Rakic, 1999; Mull and Seyal, 2001; Perry et al., 2001; Wall and Messier, 2001). Volz and colleagues began to explore the neuroanatomy of time processing by assessing patterns of brain activation during a functional magnetic resonance image (fMRI) study during which patients with schizophrenia were assessed on time estimation and pitch discrimination. Their findings, using psychophysical threshold levels, indicated that the groups differed significantly only in time estimation. In addition, their comparison of pitch versus time discrimination indicated a specific dysfunction of the right putamen, right medial/superior prefrontal cortex, and right anterior thalamus to underlie that was associated with timing dysfunction in schizophrenia. The authors speculated that, based on their findings, timing deficits in schizophrenia might be due to impairment in both the basal timing mechanisms of the basal ganglia and/or thalamus paired with impaired attentional or working memory resources that are facilitated by the prefrontal cortices. An additional finding, that is important when studying individuals with schizophrenia, is that their findings were not simply a function of the patients performing worse than the controls, as difficulty was adjusted to an individual performance level. The authors, like many to follow, argue that timing in the brain is associated with a disturbed fronto-thalamo-striatal circuit.

Davalos, Rojas, and Tregellas (2011) followed up on Volz and colleagues work with some minor changes, including varied levels of difficulty assessed between patients and controls, a larger cohort of patients and healthy comparison subjects, and the use of a $3 \mathrm{~T}$ scanner in contrast to the $1.5 \mathrm{~T}$ scanner used in the earlier study. The study not only sought to elaborate on the work of Volz and colleagues, but also to provide a follow up to the previous electrophysiological study (Davalos, Kisley, and Freedman, 2005), which indicated a dissociation between behavioral indices of time processing and neurophysiological responses to temporal deviants. Similar to the previous ERP study, participants were assessed on an "easy" and a "difficult" condition. In the "easy" condition, participants heard two tones, the first $200 \mathrm{msec}$ in duration, the second either shorter in duration (70ms, 10oms) or longer (30oms, 330ms) than the first tone. The paradigm utilized an interval, separated by 50oms. In the "difficult" condition, the duration of the second tone was more similar to the standard tone, either shorter (16oms, 17oms) or longer (230ms, 240ms; Figure 4.1).

Finally, in the base condition, both tones were 20oms. On both the "easy" and "difficult" conditions, the control group performed significantly better than the schizophrenia group. Regarding hemodynamic responses, group differences 

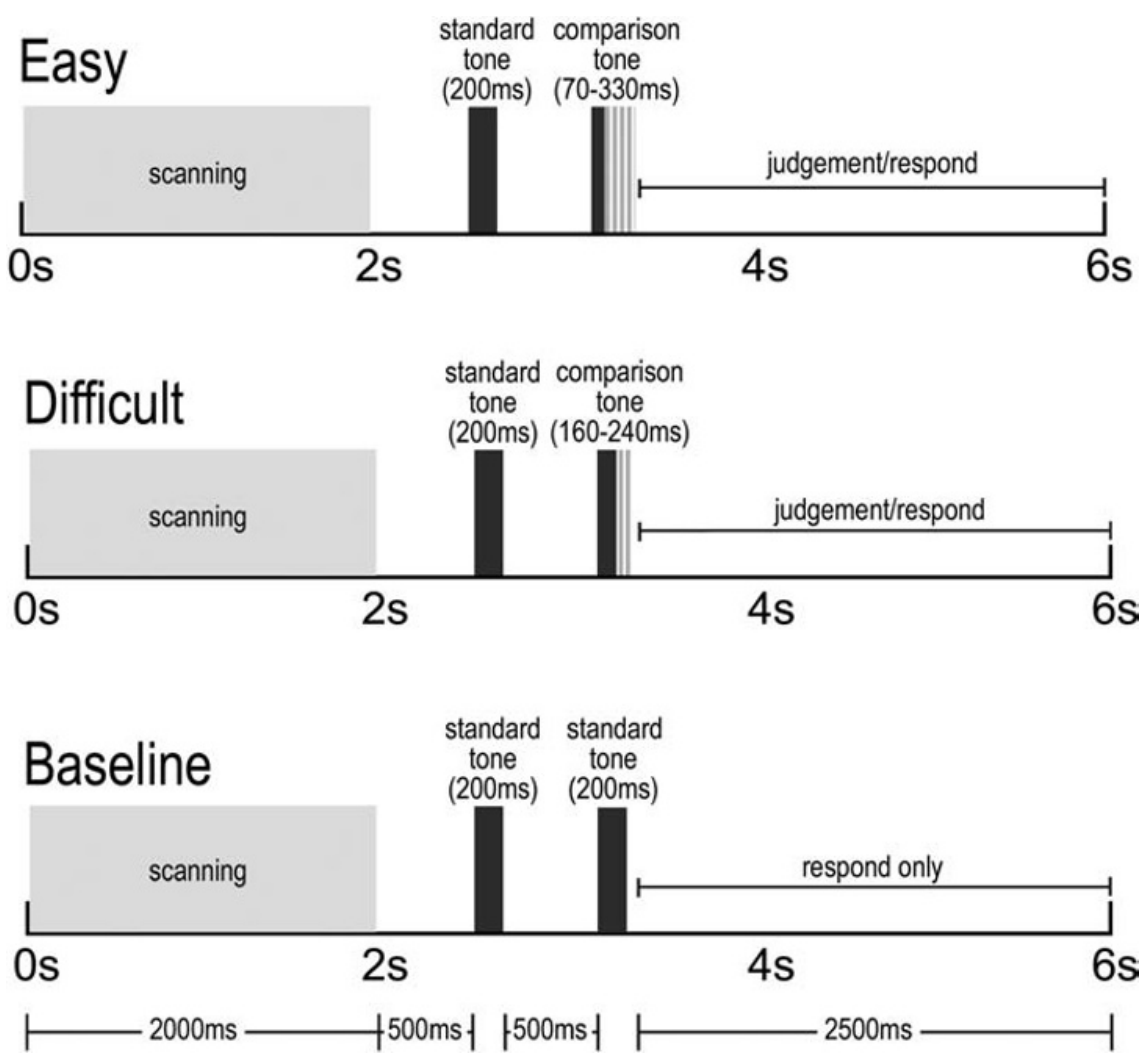

FIGURE 4.1 Schematic representation of experimental design. During the "easy" condition, the $200 \mathrm{~ms}$ standard tone was followed by a comparison tone of 70,100300 or $330 \mathrm{~ms}( \pm 50$ or $65 \%$ ). During the "difficult" condition, the comparison tone was 160, 170, 230 or $240 m s( \pm 15,20 \%)$. During “baseline," subjects pressed a button, but did not judge, following two 20oms tones. Reprinted from Schizophrenia Research, Vol. 127, D.B. Davalos, D.C. Rojas, and J.R. Tregellas, Temporal processing in schizophrenia: Effects of task-difficulty on behavioral discrimination and neuronal responses, pp. 123-130. COPYRIGHT 2011, WITH PERMISSION FROM ELSEVIER.

(control vs. schizophrenia) were assessed for three contrasts. In the easy discrimination condition, individuals with schizophrenia exhibited reduced activation in the supplementary motor area (SMA), insular/opercular cortex, and dorsolateral prefrontal cortex (DLPFC; Table 4.1, Figure 4.2).

In the "difficult" condition, the individuals with schizophrenia showed a more robust reduction in response in the SMA, insular/opercular cortex, and DLPFC. Also, reduced activation was observed in the striatum and thalamus (Figure 4.3). Regarding the difficult-easy contrast, findings indicate that the schizophrenia group exhibited reduced activation in the insular/opercular 
TABLE 4.1 MNI coordinates and statistics for brain regions with greater activation in controls compared to individuals with schizophrenia. Reprinted from Schizophrenia Research, Vol. 127, D.B. Davalos, D.C. Rojas, and J.R. Tregellas, Temporal processing in schizophrenia: Effects of task-difficulty on behavioral discrimination and neuronal responses, pp. 123-130. Copyright 2011.

\begin{tabular}{|c|c|c|c|c|c|}
\hline & $\mathbf{x}$ & $\mathbf{y}$ & $\mathbf{z}$ & t value & Pvalue \\
\hline \multicolumn{6}{|l|}{ Easy } \\
\hline Operculum/Insula (L) & -36 & 24 & -3 & 1.79 & 0.042 \\
\hline Pre-SMA (R) & 12 & 21 & $5^{1}$ & 1.69 & 0.051 \\
\hline DLPFC (L) & -48 & 9 & 36 & 1.88 & 0.035 \\
\hline \multicolumn{6}{|l|}{ Difficult } \\
\hline \multirow[t]{2}{*}{ Operculum/Insula (R) } & $5^{1}$ & 24 & o & 2.06 & 0.03 \\
\hline & 30 & 24 & -6 & 2.25 & 0.016 \\
\hline Operculum/Insula (L) & 36 & 27 & -9 & 2.69 & 0.006 \\
\hline Pre-SMA (R) & 12 & 24 & $5^{1}$ & 2.8 & 0.004 \\
\hline Putamen (R) & 18 & 12 & 6 & 1.85 & 0.37 \\
\hline Putamen (L) & -24 & -3 & 3 & 2.24 & 0.016 \\
\hline DLPFC (R) & 45 & 12 & 18 & 1.95 & 0.03 \\
\hline DLPFC (L) & -45 & 12 & 36 & 1.77 & 0.043 \\
\hline \multicolumn{6}{|l|}{ Difficult-Easy } \\
\hline Operculum/Insula (R) & 48 & 27 & 3 & 2.07 & 0.023 \\
\hline Putamen (R) & 21 & 18 & 3 & 2.46 & $>0.001$ \\
\hline Putamen (L) & -24 & 18 & -6 & 1.67 & $0.05^{2}$ \\
\hline
\end{tabular}

cortex and striatum (Figure 4.4) and striatum Ro Is. The findings described a network of brain regions with reduced temporal processing-related responses in the participants diagnosed with schizophrenia. Included in this network were the SMA, DLPFC, striatum, thalamus, and insula/operculum. Also observed were differing levels of activation, or to be precise, more pronounced differences between patients with schizophrenia and controls in striatum and insula/operculum function under conditions of high task difficulty.

What appears to be clear from neuroimaging data is that timing in the schizophrenic brain is complex. While there are similarities to healthy controls, there are clearly regions that appear to "work harder" to process temporal information. There are also regions that seem to underperform when engaged to make temporal distinctions. For example, the (pre) SMA appears to be underactive in schizophrenia, independent of task difficulty. The SMA is thought to 


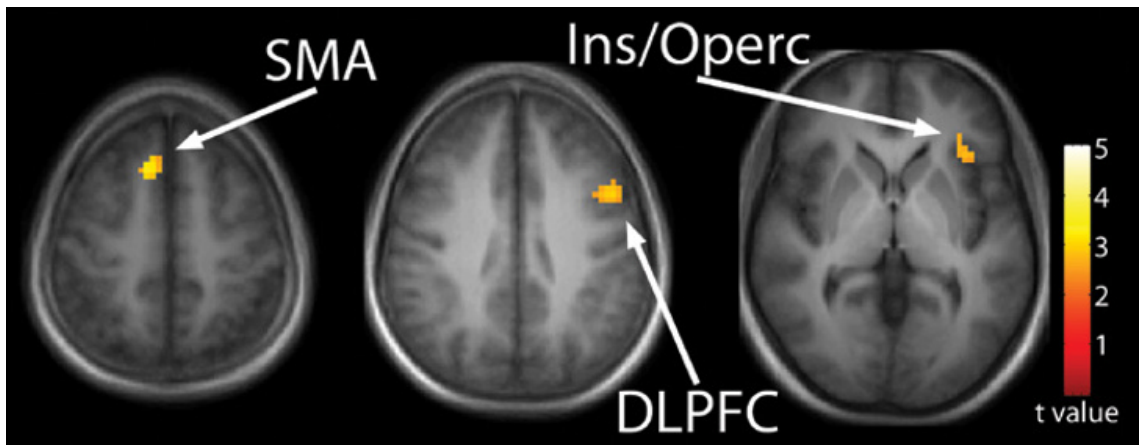

FIGURE 4.2 Temporal processing during "easy" temporal processing. Reduced activation in the schizophrenia group relative to controls was observed in the supplementary motor area (SMA), insula/ opercular cortex, and the dorsolateral prefrontal cortex (DLPFC). Statistical parametric maps thresholded at $p<0.01$, overlaid onto the average T1-weighted anatomy of all subjects. Reprinted from Schizophrenia Research, Vol. 127, D.B. Davalos, D.C. Rojas, and J.R. Tregellas, Temporal processing in schizophrenia: Effects of task-difficulty on behavioral discrimination and neuronal responses, $p p$. 123-130.

COPYRIGHT 2011, WITH PERMISSION FROM ELSEVIER.

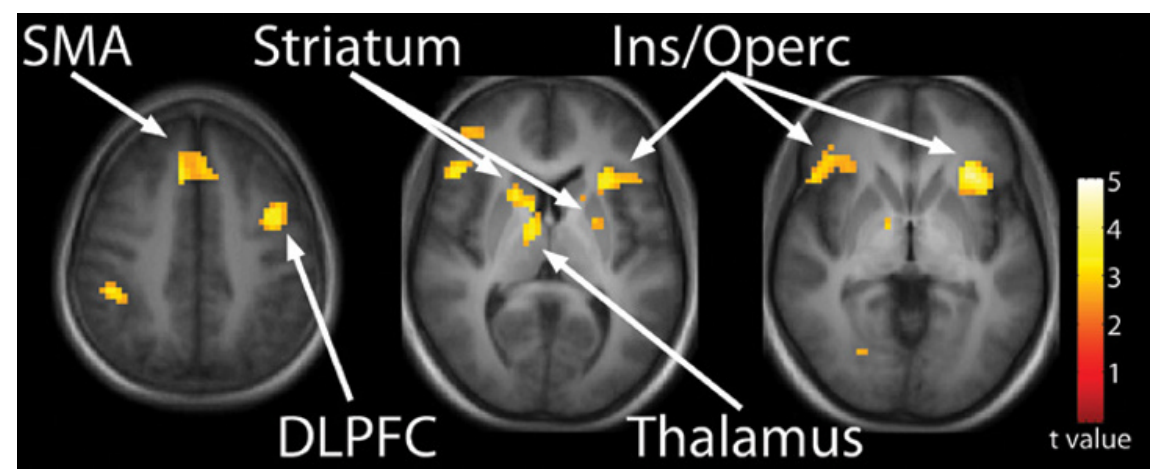

FIGURE 4.3 Temporal processing during “difficult" temporal processing. Reduced activation in the schizophrenia group relative to controls was observed in the SMA, insula/opercular cortex, and the DLPFC, as well as the striatum and thalamus. Statistical parametric maps thresholded at pbo.01, overlaid onto the average T1-weighted anatomy of all subjects. Reprinted from Schizophrenia Research, Vol. 127, D.B. Davalos, D.C. Rojas, and J.R. Tregellas, Temporal processing in schizophrenia: Effects of task-difficulty on behavioral discrimination and neuronal responses, $p p$. 123-130.

COPYRIGHT 2011, WITH PERMISSION FROM ELSEVIER.

be a key structure during temporal processing (Ferrandez et al., 2003; Macar et al., 2002; Rao et al., 2001; Tregellas et al., 2006). Specifically, the SMA has been associated with the 'pulse accumulation' process (Gibbon, Church, and Meck, 1984; Macar et al., 2004) and timing comparisons (Coull et al., 2004). Another 


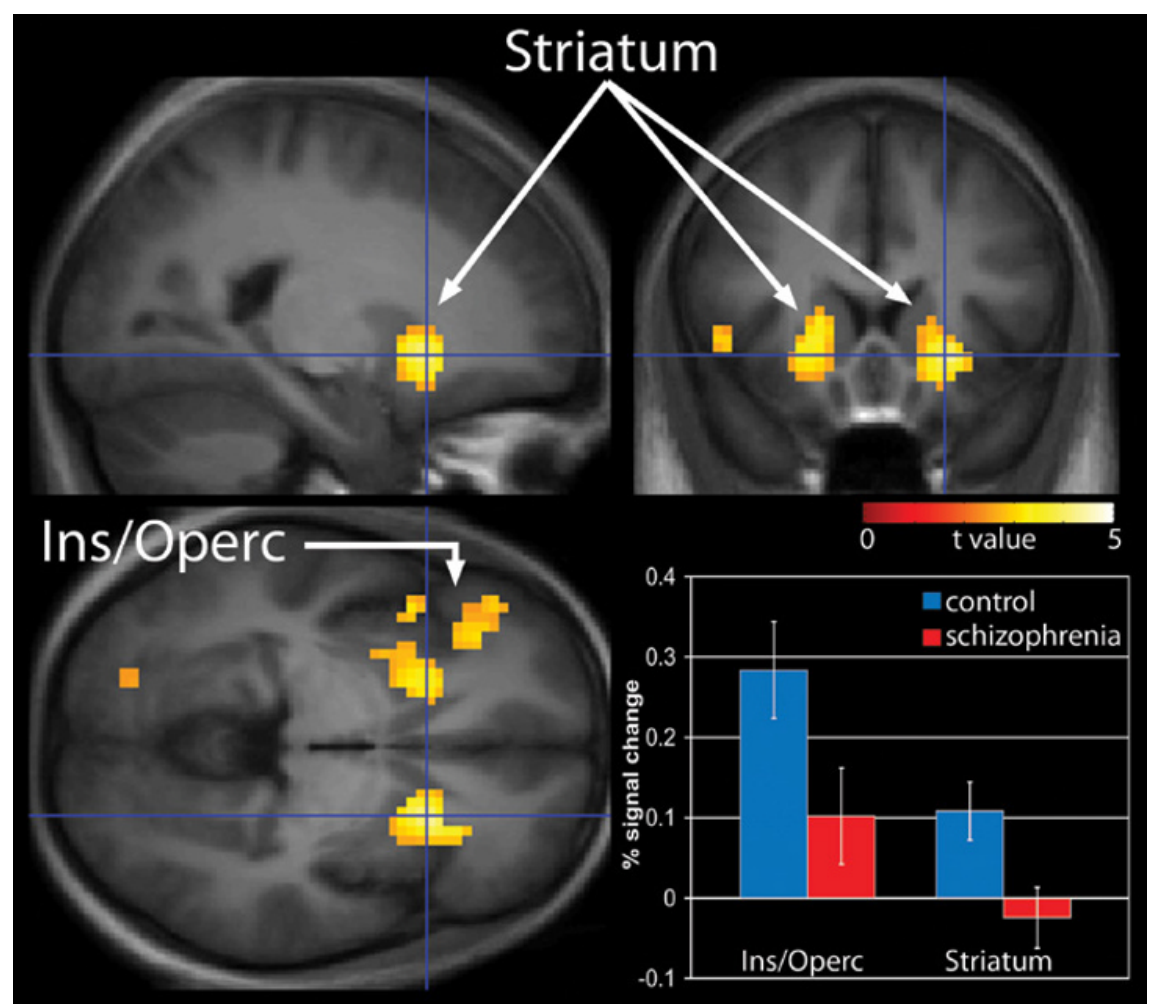

FIGURE 4.4 "Difficult" compared to "easy" temporal processing. Reduced activation in the schizophrenia group relative to controls was observed in the insula/opercular cortex and the striatum. Statistical parametric maps thresholded at pbo.o1, overlaid onto the average T1-weighted anatomy of all subjects. Reprinted from Schizophrenia Research, Vol. 127, D.B. Davalos, D.C. Rojas, and J.R. Tregellas, Temporal processing in schizophrenia: Effects of task-difficulty on behavioral discrimination and neuronal responses, pp. 123-13o. COPYRIGHT 2011, WITH PERMISSION FROM ELSEVIER.

region that appears to be underactive in schizophrenia is the insular/opercular cortices. The insular/opercular cortex has been identified in a number of timing studies and is thought to be involved in a variety of aspects of timing. These timing processes include attention to time components (Coull et al., 2000, 2004), encoding time-based sequences (Schubotz et al., 2000), and perceiving interval duration information (Hinton et al., 2004; Maquet et al., 1996; Ferrandez et al., 2003; Lewis and Miall, 2003). The DLPFC is a region of the brain that is linked to various types of cognitive deficits in schizophrenia, including higher level cognitive functioning (Glahn et al., 2005; Wilmsmeier et al., 2010). Some have speculated that the DLPFC differences observed in schizophrenia versus control studies represents a difference that emerges primarily as a function of 
task-difficulty (Manoach et al., 1999). Davalos, Rojas, and Tregellas (2011), however, found reduced activity in patients with schizophrenia regardless of task difficulty. The involvement of the DLPFC in time processing has been documented previously across a variety of timing tasks and for both healthy controls and clinical populations. DLPFC is thought to be involved in duration perception (Rao et al., 2001; Lewis and Miall, 2003; Smith et al., 2003), interval time estimation (Macar et al., 2002; Basso et al., 2003), and motor timing (Rubia et al., 1998; Jancke et al., 200o). As noted previously, there continues to be a debate regarding the role of the DLPFC in time processing. The argument persists that DLPFC may be secondarily involved in time processing, when it's primary role is associated with task difficulty or keeping information online successfully for temporal discrimination and comparison (Abi-Dargham, 2004). However, relatively recent data suggests that the DLPFC is also involved in time processing paradigms that minimize working memory components, suggesting that the reduced response in the region in schizophrenia reflects a timing-specific deficit (Davalos, Rojas, and Tregellas, 2011; Smith et al., 2003; Tregellas et al., 2006). The regions discussed so far all seem to be involved in general time processing, independent of task difficulty. All appear to be underactivated, or at least show a reduced response to timing stimuli in patients with schizophrenia compared to healthy controls. In contrast, the thalamus has been shown to be significantly diminished in schizophrenia, but only for difficult temporal distinctions (Davalos, Rojas, and Tregellas, 2011). The thalamus is one of the key structures previously identified in timing literature, both in healthy controls, and more specifically in schizophrenia. It is one of the integral structures in the fronto-striatal network that is involved in complex temporal processing tasks (e.g., perceptual timing; Menon et al., 200o; Rubia and Smith, 2004; Volz et el., 2001). Rao and colleagues' (2001) work in which thalamic responses were observed during timing, but not pitch perception, has added weight to the argument that, like the DLPFC, the thalamus plays a specific role in time processing.

Another region of the brain that has emerged as being critical in timing, and possibly associated with load-dependent temporal processing for individuals with schizophrenia is the striatum. The striatum, or more specifically the putamen, was identified as being "most impaired" in terms of the spatial extent of response differences between patients with schizophrenia and healthy controls (Davalos, Rojas, and Tregellas, 2011). The striatum has been shown to play a central role in temporal processing in animal model studies and studies assessing healthy controls compared to populations with disorders affecting the striatum (e.g., PD; Coull et al., 2004; Ferrandez et al., 2003; Harrington et al., 1998; Maricq et al., 1981; Meck, 1996; Schubotz et al., 200o). Regarding 
schizophrenia, in particular, the striatum was also identified as being a critical structure involved in temporal processing dysfunction in Volz and colleagues' (2001) work. As was described with the thalamus, timing-related responses in the striatum were reduced in the schizophrenia group only in the "difficult" condition. These findings suggests that there may be at least a couple of distinct neural networks involved in temporal processing in schizophrenia and that timing judgments that are relatively easy may result from a different system that does not involve the striatum and thalamus. It is clear that there may be core areas of the brain that are involved in timing, independent of task difficulty, while there may be other areas of the brain that are recruited for temporal processing on an as-needed basis.

In summary, it appears that the neuroanatomical basis for temporal processing deficits in schizophrenia involves a widespread network of brain regions, including the SMA, PFC, striatum, thalamus, and insula/operculum. As is consistent with behavioral and electrophysiological data, there appears to be dissociation in behavioral responses, ERP responses and activation observed in neuroimaging that is dependent on task difficulty. Specifically, difficult temporal processing paradigms appear to involve the recruitment of other cognitive processes (e.g., decision making) and supplemental brain regions (e.g., striatum and insula/operculum).

\section{4}

\section{Summary}

Eagleman (2008) questioned whether subjective time should be viewed as a unitary phenomenon, or whether there are separate neural mechanisms that typically work together, but can be dissociated under certain circumstances? Research to date suggests that there is a general temporal dysfunction in schizophrenia that appears across a variety of measures, which all loosely assess timing. What is also clear from research is that understanding and rectifying the etiology of that dysfunction is complex. We know that individuals with schizophrenia do not have only temporal processing difficulties. Schizophrenia is characterized by a variety of cognitive difficulties that may contribute to time processing deficits or simply confound our ability to truly understand timing in the schizophrenic brain. What appears fairly safe to conclude is that individuals with schizophrenia exhibit timing deficits, regardless of the method used to assess timing (e.g., behaviorally, neurophysiologically). These deficits appear to be hard-wired in some ways and appear to affect many aspects of the lives of individuals with schizophrenia. These deficits are likely exacerbated when they are combined with other demands that are deficient in this population 
(e.g., working memory, decision making) and appear to be worsened, as temporal judgments are made more difficult. It seems clear that temporal dysfunction is embedded in the psychopathology of schizophrenia and that the construct of timing needs to move up on the hierarchy of schizophrenia dysfunction. Rather than generally taking a backseat to executive dysfunction, disinhibition, and poor working memory, as it has in the past, time processing dysfunction in schizophrenia needs to be appreciated as a core deficit that may be the key to improving cognition and clinical presentation in this population.

\section{References}

Abi-Dargham, Anissa. 2004. "Do We Still Believe in the Dopamine Hypothesis? New Data Bring New Evidence" International Journal of Neuropsychopharmacology $7(\mathbf{1})$ : S1-5.

Andreasen, Nancy C. 1999. "A Unitary Model of Schizophrenia. Bleuler's 'Fragmented Phrene' as Schizencephaly" Archives of General Psychiatry 56: 781-7.

Anderson, Jonathon W., and Maureen Schmitter-Edgecombe. 2011. "Recovery of Time Estimation Following Moderate to Severe Traumatic Brain Injury" Neuropsychology 25: $36-44$.

Artieda, Julio et al. 1992. "Temporal Discrimination is Abnormal in Parkinson's Disease" Brain 115: 199-210.

Baldeweg, Torsten et al. 2002. "Impairment in Frontal But Not Temporal Components of Mismatch Negativity in Schizophrenia" International Journal of Psychophysiology 43: $111-22$.

Barabasz, Arreed F. 1973. "Temporal Orientation: A Review of the Literature" Child Study Journal Monograph (1-3): 43-9.

Basso, Gianpaolo et al. 2003. "Distributed Neural Systems for Temporal Production: A Functional MRI Study" Brain Research Bulletin 59: 405-11.

Bauermeister, Jose J. et al. 2005. "Time Estimation and Performance on Reproduction tasks in Subtypes of Children with Attention Deficit Hyperactivity Disorder" Journal of Clinical and Child Adolescent.Psychology 34: 151-62.

Carroll, Christine A. et al. 2009. "Timing Dysfunctions in Schizophrenia Span from Millisecond to Several-Second Durations" Brain and Cognition 70: 181-90.

Casini, Laurence and Richard Ivry. 1999. "Effects of Divided Attention on Time Perception in Patients with Lesions of the Cerebellum or Frontal Lobe" Neuropsychology 13(1): 10-21.

Clarke, Sean et al. 1996. "Exploring the Domain of the Cerebellar Timing System" In Maria A. Pastor and Julio Artieda (Eds.), Time, Internal Clocks and Movement. Advances in Psychology, Vol. 115, pp. 257-80. Amsterdam, Netherlands: North-Holland/Elsevier. 
Condray, Ruth. 2005. "Language Disorder in Schizophrenia as a Developmental Learning Disorder" Schizophrenia Research 73: 5-20.

Coull, Jennifer T. et al. 20oo. "Orienting Attention in Time: Behavioural and Neuroanatomical Distinction between Exogenous and Endogenous Shifts" Neuropsychologia 38: 808-19.

Coull, Jennifer T. et al. 2004. "Functional Anatomy of the Attentional Modulation of Time Estimation" Science 303: 1506-8.

Davalos, Deana B., Michael A. Kisley, and Robert Freedman. 2005. "Behavioral and Electrophysiological Indices of Temporal Processing Dysfunction in Schizophrenia" Journal of Neuropsychiatry and Clinical Neurosciences 17:517-25.

Davalos, Deana B., Michael A. Kisley, and Randall G. Ross. 2002. "Deficits in Auditory and Visual Temporal Perception in Schizophrenia" Cognitive Neuropsychiatry 7: $273-82$.

Davalos, Deana B., Michael A. Kisley, and Randall G. Ross. 2003a “Effects of Interstimulus Interval Manipulation on Auditory Temporal Processing in Schizophrenia" Brain and Cognition 52: 295-301.

Davalos, Deana B., Michael A. Kisley, and Randall G. Ross. 2003b. "Mismatch Negativity in Detection of Interval Duration Deviation in Schizophrenia" Neuroreport 14: 1283-6.

Davalos, Deana B., Donald C. Rojas, and Jason R. Tregellas. 2011. "Temporal Processing in Schizophrenia: Effects of Task-Difficulty on Behavioral Discrimination and Neuronal Responses" Schizophrenia Research 127: 123-30.

Deouell, Leon Y., Schlomo Bentin, and Marie-Hélène Giard. 1998. "Mismatch Negativity in Dichotic Listening: Evidence for Interhemispheric Differences and Multiple Generators” Psychophysiology 35: 355-65.

Eagleman David M. 2008. "Human time perception and its illusions." Current Opinion in Neurobiology 18(2): 131-6.

Eagleman, David M. 20o9. "Temporality, Empirical Approaches" In: The Oxford Companion to Consciousness. Patrick Wilken, Tim Bayne, and Axel Cleeremans (Eds). Oxford University Press: Oxford.

Eagleman, David M., and Alex O. Holcombe. 2002. "Causality and the Perception of Time" Trends in Cognitive Sciences 6: 323-5.

Eagleman, David M. et al. 2005. "Time and the Brain: How Subjective Time Relates to Neural Time" Journal of Neuroscience 25: 10369-71.

Efron, Robert. 1963. "Temporal Perception, Aphasia, and Deja Vu" Brain 86: 403-24.

Ferrandez, Anne Marie et al. 2003. "Basal Ganglia and Supplementary Motor Area Subtend Duration Perception: An fMRI Study” Neuroimage 19: 1532-44.

Fischer, F. 1929. "Zeitstruktur und Schizophrenie" Zeitschrift für die Gesamte Neurologie und Psychiatrie 121: 544-74.

Fischer, F. 1930a. "Raum-Zeit-Struktur und Denkstorung in der Schizophrenic" Zeitschrift jur die gesamte Neurologie und Psychiatrie 124: 241-56. 
Fischer, F. 1930b. "Die Zeitstorung als Schizophreniesymptom" Zentbl. f. d. ges. Neur. u. Psyckiat. 56: 455-6.

Fischer, F. 1930c. "Weitere Mitteilung uber das schizophrene Zeiterleben (zugleich ein Beitrag zum Verlaufsproblem)" Neur. u. Psychiat 57:563-4.

Frith, Chris D., Sarah-Jayne Blakemore, and Daniel M. Wolpert. 20oo. "Explaining the Symptoms of Schizophrenia: Abnormalities in the Awareness of Action" Brain Research Reviews 31: 357-63.

Gandhi S.K. et al. 2007. "Timing Judgments in Schizophrenia” (Paper Presented at the Society for Neuroscience Annual Meeting; San Diego, CA. 2007).

Gibbon, John, Russell M. Church, and Warren H. Meck. 1984. "Scalar Timing in Memory" Annals of the New York Academy of Science. 423: 52-77.

Glahn, David C. et al. 2005. "Beyond Hypofrontality: A Quantitative Meta-Analysis of Functional Neuroimaging Studies of Working Memory in Schizophrenia" Human Brain Mapping 25: 60-9.

Goldman-Rakic, Patricia S., and Lynn D. Selemon. 1997. "Functional and Anatomical Aspects of Prefrontal Pathology in Schizophrenia" Schizophrenia Bulletin 23: 437-58.

Goldman-Rakic, Patricia S. 1999. "The Physiological Approach: Functional Architecture of Working Memory and Disordered Cognition in Schizophrenia" Biological Psychiatry 46: 650-61.

Green, Michael F. 1996. "What are the Functional Consequences of Neurocognitive Deficits in Schizophrenia?" American Journal of Psychiatry 15: 321-30.

Green, Michael F. et al. 2000. "Neurocognitive Deficits and Functional Outcome in Schizophrenia: Are We Measuring the 'Right Stuff'?" Schizophrenia Bulletin 26: 119-36. Harrington, Deborah L., and Kathleen Y. Haaland. 1998. "Temporal Processing in the Basal Ganglia" Neuropsychology 12: 3-12.

Hazeltine, Eliot, Laura Helmuth, and Richard B. Ivry. 1997. "Neural Mechanisms of Timing" Trends in Cognitive Science 1: 163-9.

Head, Denise et al. 2008. "Neuroanatomical and Cognitive Mediators of Age-Related Differences in Episodic Memory" Neuropsychology 22: 491-507.

Heatherington, Ross, Maureen Dennis, and B. Spiegler. 2000. "Perception and Estimation of Time in Long-Term Survivors of Childhood Posterior Fossa Tumors" Journal of the International Neuropsychological Society 6: 682-92.

Hinton, Sean C. et al. 2004. "Neural Systems Supporting Timing and Chronometric Counting: An fMRI Study" Cognitive Brain Research 21: 183-92.

Hyde, Thomas M., James C. Ziegler, and Daniel R. Weinberger. 1993. "Psychiatric Disturbances in Metachromatic Leukodystrophy. Insights into the Neurobiology of Psychosis" Archives of Neurology 49: 401-6.

Israeli, N. 1932. "The Psychopathology of Time" Psychological Review 39(5): 486-91. Ivry, Richard B. 1996. "The Representation of Temporal Information in Perception and Motor Control" Current Opinions in Neurobiology 6: 851-7. 
Ivry, Richard B., and Steven W. Keele.1989. "Timing Functions of the Cerebellum" Journal of Cognitive Neuroscience 1: 136-51.

Jancke, Lutz et al. 2000. "Cortical Activations during Paced Finger-Tapping Applying Visual and Auditory Pacing Stimuli” Cognitive Brain Research 10: 51-66.

Kaminsky, Matti, Zohar Eviatar, and Joel Norman. 2002. "The Timing Deficit Hypothesis of Dyslexia and Its Implications for Hebrew Reading" Brain and Cognition 48: 394-8.

Kisley, Michael A. et al. 2004. "Small Changes in Temporal Deviance Modulate Mismatch Negativity Amplitude" Neuroscience Letters 358: 197-200.

Kraus, Nina. 2003. "Auditory Pathway Encoding and Neural Plasticity in Children with Learning Problems" Audiology and Neurotology 6: 221-7.

Lehmann, Heinz E. 1967. "Time and Psychopathology" Annals of the New York Academy of Sciences 138: 798-821.

Lewis, Penny A., and R. Chris Miall. 2003. "Brain Activation Patterns During Measurement of Sub- and Supra-Second Intervals" Neuropsychologia 41: 1583-92.

Light, Gregory A., and David L. Braff. 2005a. "Mismatch Negativity Deficits are Associated with Poor Functioning in Schizophrenia Patients" Archives of General Psychiatry 62: 127-36.

Light, Gregory A., and David L. Braff. 2005b. "Stability of Mismatch Negativity Deficits and Their Relationship to Functional Impairments in Chronic Schizophrenia" American Journal of Psychiatry 162: 1741-3.

Macar, Francois, and Franck Vidal. 2009. "Timing Processes: An Outline of Behavioral and Neural Indices Not Systematically Considered in Timing Models" Canadian Journal of Experimental Psychology 63: 227-39.

Macar, Francois, Jennifer Coull, and Franck Vidal. 2006. "The Supplementary Area in Motor and Perceptual Time Processing: fMRI Studies" Cognitive Processing 7: 89-94.

Macar, Francois et al. 2002. "Activation of the Supplementary Motor Area and of Attentional Networks during Temporal Processing” Experimental Brain Research 142: $475^{-85}$.

Macar, Francois et al. 2004. "Timing Functions of the Supplementary Motor Area: An Event-Related fMRI Study" Cognitive Brain Research 21: 206-15.

Maquet, Pierre et al. 1996. "Brain Activation Induced by Estimation of Duration: A PET Study” Neuroimage 3: 119-26.

Mangels, Jennifer A., Richard Ivry, and Naomi Shimizu. 1998. "Dissociable Contributions of the Prefrontal and Neocerebellar Cortex to Time Perception" Cognitive Brain Research 7: 15-39.

Manoach, Dara S. et al. 1999. "Schizophrenic Subjects Activate Dorsolateral Prefrontal Cortex during a Working Memory Task, as Measured by fMRI" Biological Psychiatry 45: 1128-37. 
Maricq, Andres V., S. Roberts, and Russell M. Church. 1981. "Methamphetamine and Time Estimation" Journal of Experimental Psychology; Animal Behavior 7: 18-30.

McGlashan, Thomas H., and Ralph E. Hoffman. 2000. "Schizophrenia as a Disorder of Developmentally Reduced Synaptic Connectivity." Archives of General Psychiatry 57: 637-48.

Meck, Warren H. 1996. "Neuropharmacology of Timing and Time Perception" Cognitive Brain Research 3: 227-42.

Menon, Vinod et al. 2000. "Basal Ganglia Involvement in Memory-Guided Movement Sequencing" Neuroreport 11: $3641-5$.

Merzenich Michael M. et al. 1996. “Temporal Processing Deficits of Language-Learning Impaired Children Ameliorated by Training” Science 271: 77-81.

Michie Patricia T. et al. 2002. "Duration Mismatch Negativity in Biological Relatives of Patients with Schizophrenia Spectrum Disorders" Biological Psychiatry 52: 749-58.

Minkowski, E. 1928. "Quelques remarques sur la psychopathologie de la demence senile" Journal de Psychologie 25: 79-90.

Minkowski, E. 1931. "Das Zeit- und das Raumproblem in der Psychopathologie" Wiener klinische Wochenschrift, 44: 380-4.

Mull, B.R., and Masud Seyal. 2001. "Transcranial Magnetic Stimulation of Left Prefrontal Cortex Impairs Working Memory" Clinical Neurophysiology 112: 1672-5.

Naatanen, Risto. 1992. Attention and Brain Function. Hillsdale, NJ, Erlbaum.

Naatanen, Risto et al. 1993. "Event-Related Potentials Reveal a Memory Trace for Temporal Features" Neuroreport 5: 310-2.

Navon, David. 1978. "On a Conceptual Hierarchy of Time, Space, and Other Dimensions" Cognition 6: 223-8.

Nichelli, Paolo et al. 1995. "Duration Processing after Frontal Lobe Lesions" In Jordan Grafman, Keith J. Holyoak, and F. Boller (Eds.), Annals of the New York Academy Sciences (Vol. 769, pp. 183-90). New York: New York Academy of Sciences.

Palmer, Barton W. et al. 2002. "Heterogeneity in Functional Status Among Older Outpatients with Schizophrenia: Employment History, Living Situation, and Driving" Schizophrenia Research 55: 205-15.

Perry, William et al. 2001. "Working Memory in Schizophrenia: Transient "Online" Storage Versus Executive Functioning” Schizophrenia Bulletin 27(1): 157-76.

Poynter, W. Douglas, and Donald Homa. 1983. "Duration Judgment and the Experience of Change" Perception and Psychophysics 33: 549-6o.

Pulvermuller, Friedemann, and Yury Shtyrov. 2003. "Automatic Processing of Grammar in the Human Brain as Revealed by the Mismatch Negativity" Neuroimage 20: 159-72.

Rabin, A.I. 1957. "Time Estimation of Schizophrenics and Non-Psychotics" Journal of Clinical Psychology 13: 88-90.

Rammsayer, Thomas. 1990. "Temporal Discrimination in Schizophrenic and Affective Disorders: Evidence for a Dopamine-Dependent Internal Clock" International Journal of Neuroscience 53: 111-20. 
Rammsayer, Thomas. 1993. "On Dopaminergic Modulation of Temporal Information Processing" Biological Psychiatry 36: 209-22.

Rao, Stephen M., Andrew R. Mayer, and Deborah L. Harrington. 2001. "The Evolution of Brain Activation during Temporal Processing" Nature Neuroscience 4: 317-23.

Rubia, Katya, and Anna Smith. 2004. "The Neural Correlates of Cognitive Time Management: A Review" Acta Neurobiologiae Experimentalis 64: 329-40.

Rubia, Katya et al. 1998. "Prefrontal Involvement in "Temporal Bridging" and Timing Movement" Neuropsychologia 36: 1283-93.

Rueda, Alicia D., and Maureen Schmitter-Edgecombe. 20o9. "Time Estimation Abilities in Mild Cognitive Impairment and Alzheimer's Disease" Neuropsychology 23: $178-88$.

Salisbury, Dean F. et al. 2002a. "Mismatch Negativity in Chronic Schizophrenia and First-Episode Schizophrenia" Archives of General Psychiatry 59: 686-94.

Salisbury, Dean F. et al. 2002b. "Semantic Bias, Homograph Comprehension, and EventRelated Potentials in Schizophrenia" Clinical Neurophysiology 113: 383-95.

Salthouse, Timothy 1985. "A. Speed of Behavior and Its Implications for Cognition" In James E. Birren and K. Warner Schaie (Eds.), Handbook of the psychology of aging (pp. 400-26). New York: Van Nostrand Reinhold.

Schilder, Paul. 1936. "Psychopathology of Time" The Journal of Nervous and Mental Disorders 83: 530-46.

Schubotz, Ricarda I., Angela D. Friederici, and D. Yves von Cramon. 20oo. "Time Perception and Motor Timing: A Common Cortical and Subcortical Basis Revealed by fMRI" Neuroimage 11: 1-12.

Schwartz, Barry D., David B. Mallott, and Daniel K. Winstead. 1988. "Preattentive Deficit in Temporal Processing by Chronic Schizophrenics" Biological Psychiatry 23: 664-9.

Smith, Anna et al. 2003. "A Right Hemispheric Frontocerebellar Network for Time Discrimination of Several Hundreds of Milliseconds" Neuroimage 20:344-50.

Stetson, Chess et al. 2006. "Motor-Sensory Recalibration Leads to an Illusory Reversal of Action and Sensation" Neuron 51: 651-9.

Sussman, Elyse, István Winkler, and Wenjung Wang. 2003. "MMN and Attention: Competition for Deviance Detection" Psychophysiology 40: 430-35.

Tervaniemi, Mari et al. 1999. "Test-Retest Reliability of Mismatch Negativity for Duration, Frequency and Intensity Changes" Clinical Neurophysiology 110(8): 1388-93.

Todd, Juanita, Patricia T. Michie, and Assen V. Jablensky. 2003. "Association between Reduced Duration Mismatch Negativity (MMN) and Raised Discrimination Thresholds in Schizophrenia" Clinical Neurophysiology 114: 2061-70.

Tracy, Joseph I. et al. 1998. "Information-Processing Characteristics of Explicit Time Estimation by Patients with Schizophrenia and Normal Controls" Perceptual and Motor Skills 86: 515-26. 
Tregellas, Jason R., Deana B. Davalos, and Donald C. Rojas. 2006. "Effect of Task Difficulty on the Functional Neuroanatomy of Temporal Processing" Neuroimage 32: 307-15.

Tysk, Lennart. 1990. "Estimation of Time by Patients with Positive and Negative Schizophrenia." Perceptual and Motor Skills 71: 826.

Umbricht, Daniel et al. 2003. "How Specific are Deficits in Mismatch Negativity Generation to Schizophrenia?" Biological Psychiatry 53: 1120-31.

Verhaeghen, Paul, and Timothy A. Salthouse. 1997. "Meta-Analyses of Age-Cognition Relations in Adulthood: Estimates of Linear and Nonlinear Age Effects and Structural Models" Psychological Bulletin 122: 231-49.

Volz, Hans-Peter et al. 2001. "Time Estimation in Schizophrenia: An fMRI Study at Adjusted Levels of Difficulty" NeuroReport 12: 313-6.

Wall, Philip M., and Claude Messier. 2001. "The Hippocampal Formation-Orbitomedial Prefrontal Cortex Circuit in the Attentional Control of Active Memory" Behavioural Brain Research 127: 99-117.

Wallace, Gregory L., and Francesca Happe. 2008. "Time Perception in Autism Spectrum Disorders" Research in Autism Spectrum Disorders (2): 447-55.

Wilmsmeier, Andreas et al. 2010. "Neural Correlates of Set-Shifting: Decomposing Executive Functions in Schizophrenia" Journal of Psychiatry and Neuroscience 35(5): $321-9$.

Zacks, Rose T., and Lynn Hasher. 1994. "Directed Ignoring: Inhibitory Regulation of Working Memory" In Dale Dagenbach and Thomas H. Carr (Eds.), Inhibitory Processes in Attention, Memory, and Language. (pp. 241-64). San Diego, CA: Academic Press.

Ziegler, Wolfram, and D. Yves Von Cramon. 1986. "Disturbed Coarticulation in Apraxia of Speech: Acoustic Evidence" Brain and Language 29: 34-47. 